\title{
The Art of Femtosecond Laser Writing
}

\author{
Peter G. Kazansky and Weijia Yang \\ Optoelectronics Research Centre, University of Southampton, SO17 1BJ, United Kingdom \\ pgk@orc.soton.ac.uk \\ Yasuhiko Shimotsuma and Kazuyuki Hirao \\ Department of Material Chemistry, Graduate School of Engineering, Kyoto University, Kyoto, Japan 615-8510 \\ Alan Arai \\ Applications Research Laboratory, IMRA America, Inc., Fremont, CA 94538, USA \\ Yuri P. Svirko \\ Department of Physics and Mathematics, University of Joensuu, FI-80101, Finland
}

\begin{abstract}
Common beliefs that laser writing does not change when reversing beam scan or propagation direction are challenged. Recently discovered phenomena of quill and non-reciprocal femtosecond laser writing in glasses and crystals are reviewed.
\end{abstract}

(C) 2009 Optical Society of America

OCIS codes (140.3390) Laser materials processing, (320.7120) Ultrafast phenomena

Modification of transparent materials with ultrafast lasers has attracted considerable interest due to a wide range of applications including laser surgery, integrated optics, optical data storage, 3D microand nano-structuring [1].Three different types of material modifications can be induced with ultrafast laser irradiation in the bulk of a transparent material, silica glass in particular: an isotropic refractive index change (type 1); a form birefringence associated with self-assembled nanogratings and negative refractive index change (type 2) [2,3]; and a void (type 3). In fused silica the transition from type 1 to type 2 and finally to type 3 modification is observed with an increase of fluence.

Recently, a remarkable phenomenon in ultrafast laser processing of transparent materials has been reported manifesting itself as a change in material modification by reversing the writing direction

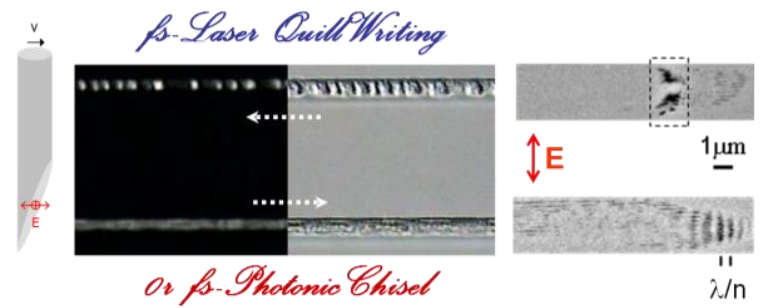

Fig. 1 (centre) Bright field images (light part) and images in crossed polarizers (dark part) of the lines written in opposite directions with amplified $\mathrm{Yb}$ fiber laser at $500 \mathrm{kHz}$ repetition rate, writing speed $250 \mu \mathrm{m} / \mathrm{s}$ and pulse energy $0.9 \mu \mathrm{J}$. (left)The tilted front of the pulse along writing direction is shown. (right) SEM images of cross sections of lines written with polarization perpendicular to writing direction are also shown. The nanograting of about $300 \mathrm{~nm}$ period, which is responsible for the form birefringence of irradiated regions, can be seen only in the initial part of cross sections of lines written in one of two directions. The region of collateral damage is marked with dashed line.

(Fig.1) [4]. The phenomenon has been interpreted in terms of anisotropic plasma trapping and heating by a tilted front of the ultrashort laser pulse.

More recently, we have experimentally demonstrated that indeed the pulse front tilt [5] can be used to control material modifications and in particular as a new tool for laser processing and optical manipulation, e.g. for achieving calligraphic-style laser writing, when the appearance of a "stroke" varies in relation to its direction (Fig. 2) [6].
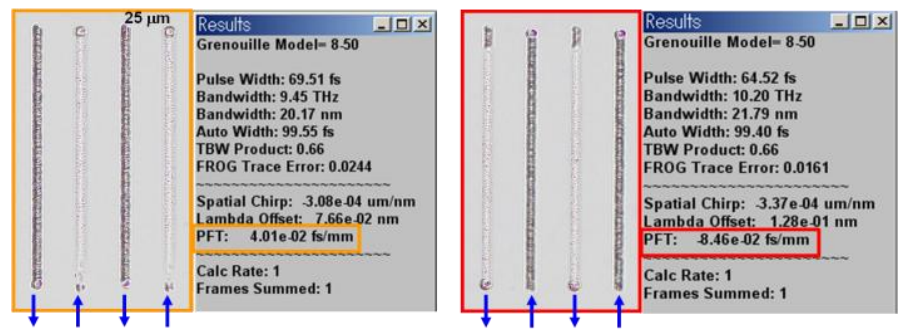

Fig. 2 Microscope bright-field image of the line structures written with a Ti:sapphire laser at $800 \mathrm{~nm}, 250 \mathrm{kHz}, 50 \mu \mathrm{m} / \mathrm{s}$ using pulses with (left) positive pulse front tilt or (right) negative pulse front tilt. The writing direction is shown by the arrows. The corresponding screen shots containing measured laser pulse parameters by a GRENOUILLE device are shown. 
Moreover, a new type of light-induced modification in a solid, namely an anisotropic cavitation, was observed in the vicinity of the focus at high fluences (Fig. 3). The bubbles can be trapped and manipulated in the plane perpendicular to the light propagation direction by controlling the laser

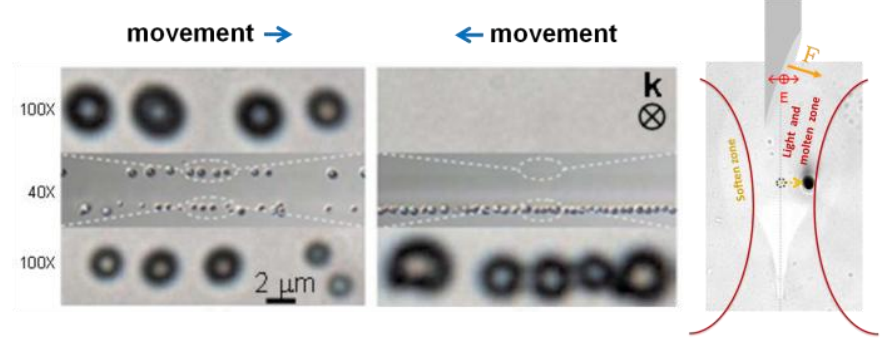

Fig. 3 Microscope images with different magnifications of the line structures fabricated in opposite directions with $2.4 \mu \mathrm{J}$ pulse energy and scan speed of $50 \mu \mathrm{m} / \mathrm{s}$ (left and center) and a cross-section of the line structure (right).

writing direction relative to the tilt of the pulse front. It should be pointed out that since the discovery of lasers, it has been believed that interactions of a Gaussian laser beam with an isotropic medium can produce only centrosymmetric material modifications. Our experiments provide the evidence that it is not always true.

It has also been a common belief that in a homogeneous medium, the photosensitivity and corresponding light-induced material modifications do not change on the reversal of light propagation direction. Recently, we have demonstrated that when the direction of the femtosecond laser beam is reversed from $+\mathrm{Z}$ to $-\mathrm{Z}$ directions, the structures written in a lithium niobate crystal are mirror images
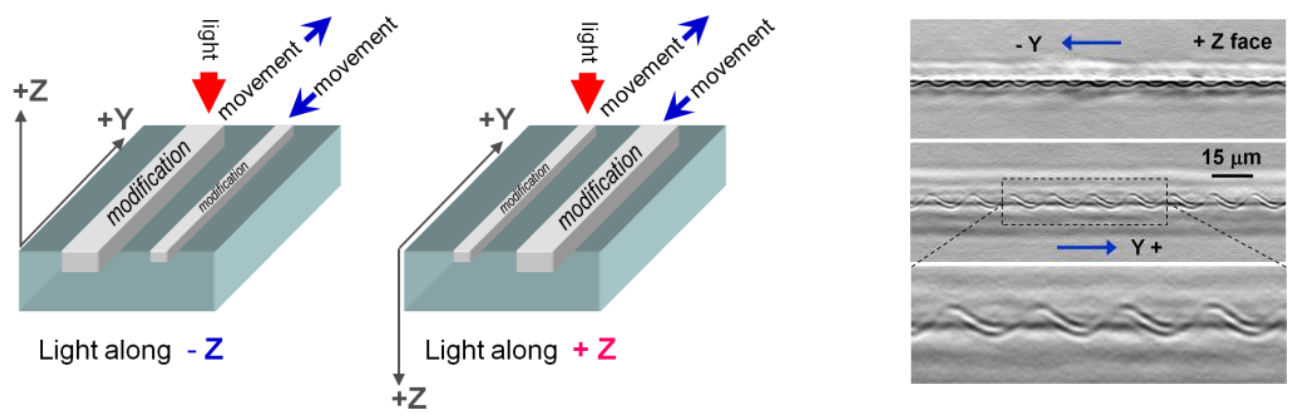

Fig. 4 The illustration of non-reciprocal laser writing - KaYaSo effect ((left and centre) and DIC images of wavy selforganized structures created when writing along the $-\mathrm{Y}$ axis and the $+\mathrm{Y}$ axis in lithium niobate (right).

when translating the beam along the $+Y$ and $-Y$ directions (Fig. 4) [7]. In contrast to glass, the directional dependence of writing in lithium niobate depends on the orientation of the crystal with respect to the direction of the beam movement and the light propagation direction. We demonstrate theoretically that interplay of the crystal anisotropy and light-induced heat flow gives rise to a new nonreciprocal nonlinear optical phenomenon, nonreciprocal photosensitivity. In the lithium niobate, the nonreciprocal photosensitivity manifests itself as a changing the sign of the light-induced current when the light propagation direction is reversed. Therefore, in a non-centrosymmetric medium, modification of the material can be different when light propagates in opposite directions. Non-reciprocity is produced by magnetic field (Faraday effect) and movement of the medium with respect to the direction of light propagation: parallel (Sagnac effect) or perpendicular (KaYaSo effect).

We anticipate that the observed phenomena will open new opportunities in laser material processing, laser surgery, optical manipulation and data storage.

\section{References}

[1] R. R. Gattas and E. Mazur, "Femtosecond laser micromachining in transparent materials," Nature Photonics 2, 219-225 (2008).

[2] Y. Shimotsuma, P. G. Kazansky, J. Qiu and K. Hirao, "Self-organized nanogratings in glass irradiated by ultrashort light pulses," Phys. Rev. Lett. 91, 247705 (2003).

[3] V. Bhardwaj, E. Simova, P. Rajeev, C. Hnatovsky, R. Taylor, D. Rayner and P. Corkum, "Optically produced arrays of planar nanostructures inside fused silica," Phys. Rev. Lett. 96, 057404-1 (2006).

[4] P. G. Kazansky, W. Yang, E. Bricchi, J. Bovatsek, A. Arai, Y. Shimotsuma, K. Miura and K. Hirao,"Quill" writing with ultrashort light pulses in transparent materials," Appl. Phys. Lett. 90, 151120 (2007).

[5] S. Akturk, M. Kimmel, P. O'Shea, and R. Trebino, "Measuring spatial chirp in ultrashort pulses using single-shot FrequencyResolved Optical Gating,” Opt. Express 11, 68-78 (2003).

[6] W. Yang, P. G. Kazansky, Y. Shimotsuma, M. Sakakura, K. Miura and K. Hirao, “Ultrashort-pulse laser calligraphy,” Appl. Phys. Lett. 93, 171109 (2008).

[7] W. Yang, P. G. Kazansky and Yu. P. Svirko, “Non-reciprocal ultrafast laser writing,” Nature Photonics, 2, 99-105 (2008). 